\title{
The nuclear matrix protein HNRNPU maintains 3D genome architecture globally in mouse hepatocytes
}

\author{
Hui Fan, ${ }^{1,6}$ Pin Lv, ${ }^{1,6}$ Xiangru Huo, ${ }^{1}$ Jicheng Wu, ${ }^{1}$ Qianfeng Wang, ${ }^{1}$ Lu Cheng, ${ }^{1}$ \\ Yun Liu, ${ }^{1}$ Qi-Qun Tang, ${ }^{1}$ Ling Zhang, ${ }^{2}$ Feng Zhang, ${ }^{2,3}$ Xiaoqi Zheng, ${ }^{4} \mathrm{Hao} \mathrm{Wu},{ }^{5}$ \\ and Bo Wen ${ }^{1,3}$

\begin{abstract}
${ }^{1}$ MOE Key Laboratory of Metabolism and Molecular Medicine, Institutes of Biomedical Sciences, and Department of Biochemistry and Molecular Biology, Fudan University Shanghai Medical College, Shanghai 200032, China; ${ }^{2}$ Obstetrics and Gynecology Hospital, Fudan University, Shanghai 200011, China; ${ }^{3}$ State Key Laboratory of Genetic Engineering, Collaborative Innovation Center of Genetics and Development, Fudan University, Shanghai 200438, China; ${ }^{4}$ Department of Mathematics, Shanghai Normal University, Shanghai 200234, China; ${ }^{5}$ Department of Biostatistics and Bioinformatics, Rollins School of Public Health, Emory University, Atlanta, Georgia 30322, USA
\end{abstract}

\begin{abstract}
Eukaryotic chromosomes are folded into higher-order conformations to coordinate genome functions. In addition to longrange chromatin loops, recent chromosome conformation capture (3C)-based studies have indicated higher levels of chromatin structures including compartments and topologically associating domains (TADs), which may serve as units of genome organization and functions. However, the molecular machinery underlying these hierarchically three-dimensional (3D) chromatin architectures remains poorly understood. Via high-throughput assays, including in situ Hi-C, DamID, ChIP-seq, and RNA-seq, we investigated roles of the Heterogeneous Nuclear Ribonucleoprotein U (HNRNPU), a nuclear matrix (NM)-associated protein, in 3D genome organization. Upon the depletion of HNRNPU in mouse hepatocytes, the coverage of lamina-associated domains (LADs) in the genome increases from $53.1 \%$ to $68.6 \%$, and a global condensation of chromatin was observed. Furthermore, disruption of HNRNPU leads to compartment switching on $7.5 \%$ of the genome, decreases TAD boundary strengths at borders between A (active) and B (inactive) compartments, and reduces chromatin loop intensities. Long-range chromatin interactions between and within compartments or TADs are also significantly remodeled upon HNRNPU depletion. Intriguingly, HNRNPU mainly associates with active chromatin, and $80 \%$ of HNRNPU peaks coincide with the binding of CTCF or RAD21. Collectively, we demonstrated that HNRNPU functions as a major factor maintaining 3D chromatin architecture, suggesting important roles of NM-associated proteins in genome organization.
\end{abstract}

[Supplemental material is available for this article.]

In the eukaryotic cell nucleus, appropriate higher-order chromatin structures are critical to genome organization and functions (Cremer and Cremer 2010; Bickmore 2013; Dekker and Misteli 2015; Rowley and Corces 2016). It has been well established that long-range chromatin loops are important for connecting enhancers and promoters and for insulating chromatin domains (Tang et al. 2015; Ghirlando and Felsenfeld 2016; Hnisz et al. 2016). Furthermore, recent 3C-based experiments revealed that the genomes consist of multiple levels of self-associating chromatin structures including compartments (Lieberman-Aiden et al. 2009) and topologically associating domains (TADs) (Dixon et al. 2012; Nora et al. 2012; Sexton et al. 2012). There are two types of compartments: $\mathrm{A}$ and $\mathrm{B}$, associated with active and inactive chromatin, respectively. The compartments are recently validated as relatively stable physical structures of individual cells as detected by super-resolution microscope (Wang et al. 2016b) and single cell Hi-C (Stevens et al. 2017). TADs are evolutionarily conserved and are relatively invariant among cell types (Dixon et al. 2015; Schmitt et al. 2016). It is increasingly evident that TADs may be functional units of the genome, involving biological processes

\footnotetext{
${ }^{6}$ These authors contributed equally to this work. Corresponding author: bowen75@fudan.edu.cn Article published online before print. Article, supplemental material, and publication date are at http://www.genome.org/cgi/doi/10.1101/gr.224576.117.
}

such as transcriptional coregulation, DNA replication, and V(D)J recombination (Nora et al. 2012; Lucas et al. 2014; Pope et al. 2014; Symmons et al. 2014; Hu et al. 2015; Dekker and Mirny 2016; Dixon et al. 2016). Disruption of TADs can cause impaired development and/or disease (Groschel et al. 2014; Lupianez et al. 2015; Flavahan et al. 2016; Franke et al. 2016).

The formation and maintenance of higher-order chromatin structure require architectural proteins including the CCCTCbinding factor (CTCF) and cohesin (Gomez-Diaz and Corces 2014). The binding of CTCF is enriched at the boundary of TADs in a convergent manner (Dixon et al. 2012; Rao et al. 2014; Vietri Rudan et al. 2015). Deletion or inversion of CTCF binding sites disrupts the topology of chromatin loops (Guo et al. 2015; Narendra et al. 2015), and an extrusion model was proposed to explain the mechanism for loop formation mediated by CTCF and cohesin (Sanborn et al. 2015; Fudenberg et al. 2016). Furthermore, loss-offunction studies indicated that the depletion of CTCF or cohesin led to alterations of intra- and inter-TAD chromatin interactions (Seitan et al. 2013; Sofueva et al. 2013; Zuin et al. 2014). Other proteins such as ZNF143, SMARCA4, and RUNX1 are also found national), as described at http://creativecommons.org/licenses/by-nc/4.0/. 
to play roles in the regulation of chromatin interactions (Heidari et al. 2014; Bailey et al. 2015; Barutcu et al. 2016a,b). However, the molecular machinery responsible for global and hierarchical chromatin organization remains poorly understood.

The nuclear matrix (NM) consists of the inner NM and peripheral NM, and the latter includes nuclear lamina and nuclear pore complexes (Linnemann and Krawetz 2009). It has long been speculated that the NM provides structural frameworks for topologically confined chromatin domains (Getzenberg et al. 1991), but the roles of the inner NM in global chromatin architecture such as compartments and TADs have not been investigated systematically. As one of the major NM-associated proteins, HNRNPU (also known as SAF-A) was recognized as an attachment factor linking specific DNA elements, scaffold/matrix attachment regions (S/MAR), to the NM (Gohring and Fackelmayer 1997). Purified HNRNPU proteins can bind DNA to form higher-ordered nucleic-acid-protein complexes in vitro (Fackelmayer et al. 1994). Moreover, HNRNPU is required for the localization of Xist RNA (Hasegawa et al. 2010), the key regulator of X Chromosome inactivation. Therefore, we hypothesized that HNRNPU could play a general role in regulating global 3D genome organization.

\section{Results}

\section{HNRNPU is required for global nuclear organization}

To test roles of HNRNPU in nuclear organization, we knocked down Hnrnpu gene expression by RNA interference (RNAi) with two independent short hairpin RNAs (shRNAs) in AML12 cells (Fig. 1A), a mouse hepatocyte cell line in which the nuclear architecture is well preserved (McDonald et al. 2011; Fu et al. 2015). Based on immunofluorescence analysis, we observed strong signal reductions of the nucleolus marker $\mathrm{C} 23$ and a significant increase of heterochromatin foci numbers (marked by $\mathrm{H} 3 \mathrm{~K} 9 \mathrm{me} 3$ ) in HNRNPU-depleted cells (Fig. 1B,C). Importantly, these phenotypes can be rescued by re-expression of shRNA (shU-1)-resistant Hnrnpu cDNA, which ruled out potential off-target effects of the RNAi experiments. Total protein levels of C23 and H3K9me3 remain unchanged (Fig. 1D), suggesting a global re-organization of chromatin organization with HNRNPU depletion. Moreover, neither apparent changes of cell cycle nor signatures of cellular senescence were detected in HNRNPU-depleted cells (Supplemental Fig. $\mathrm{S} 1$ ), indicating that these alterations of nuclear organization are not due to biased cell cycle or senescence.

We then conducted DNase I digestion experiments to measure global chromatin structure. Intriguingly, HNRNPU-depleted cells display lower chromatin accessibility in mouse hepatocytes (AML12) (Fig. 1E) and human liver carcinoma cells (HepG2) (Fig. $1 F)$. These data suggest an evolutionarily conserved role of HNRNPU in maintaining global chromatin structure.

Subsequently, we applied genomic approaches to decipher functional roles of HNRNPU genome-wide. As phenotypes resulted from the knockdown by shRNA1 (shU-1) can be rescued via the re-expression of shU-1 resistant Hnrnpu cDNA (Fig. 1B,C), we only used shU-1 for further high-throughput assays (labeled as "shU"). For genome-wide experiments, we conducted two replicates using AML12 cells treated with shU-1 containing lentivirus separately.

\section{Disruption of HNRNPU alters gene expression and increases chromatin-lamina interactions}

To reveal biological consequences of HNRNPU depletion, we examined gene expression by RNA-seq. We observed significant expression changes on 639 genes upon HNRNPU depletion. Among them, 500 (78\%) genes were down-regulated and 139 genes (22\%) were up-regulated (Supplemental Table S1; Supplemental Fig. S2A,B). Gene Ontology (GO) analysis shows that upregulated genes are enriched in biological processes including cell adhesion and cell proliferation, and down-regulated genes associate with immune processes, oxidation-reduction processes, and triglyceride metabolic processes (Supplemental Fig. S2C).

To investigate spatial organization of the genome with HNRNPU depletion, we mapped chromatin-lamina interactions by DNA Adenine Methyltransferase Identification (DamID) assay with lamin B1-Dam fusion proteins. The data show that chromatin-lamina interactions are substantially remodeled upon HNRNPU depletion (Fig. 2A,B), and the genome coverage of lamina-associated chromatin domains (LADs) (Guelen et al. 2008) is increased from $53.1 \%$ to $68.6 \%$ (Fig. 2C; Supplemental Table S2). Importantly, the gain of LADs is significantly associated with the reduction of gene expression (Fig. 2D). Consistently, the electron microscope (EM) imaging displays a global relocalization of chromatin toward the nuclear membrane in HNRNPU-depleted cells (Fig. 2E). Thus, HNRNPU depletion results in spatial changes of chromatin toward the nuclear periphery, which associates with gene expression down-regulation.

\section{HNRNPU regulates chromatin compartments}

To investigate the impact of HNRNPU on chromatin interactions, we generated high-resolution interaction maps by an in situ Hi-C assay (Rao et al. 2014). We conducted two replicates of Hi-C experiments, and the results are highly consistent (Supplemental Fig. S3). Hi-C data provide information about multiscale chromatin interactions including compartments, TADs, and chromatin loops, and we first examined compartments. While contact maps of control and HNRNPU-depleted cells are similar at the chromosome level (Fig. 3A), interactions among compartments are changed globally (Fig. 3B). We applied different statistical methods to detect these differences and obtained similar results (Supplemental Fig. S4).

To take a closer look at compartment organization, we examined inter- and intra-compartment interactions. Consistent with the results of Figure 3B, inter-compartment interactions between $A$ and $B$ increase significantly, but those among the same type of compartments (A vs. A; B vs. B) decrease significantly (Fig. 3C). Further, we compared changes in inter-compartment interactions along distances. Interactions among the same type of compartments become significantly decreased if distances are $>2 \mathrm{Mb}$. However, interactions between A and B compartments decrease significantly in all distance ranges (Supplemental Fig. S5). Moreover, intra-compartment interactions significantly increase in A compartments but decrease in B compartments (Fig. 3D). Of note, results obtained from replicates (rep1 and rep2) are almost identical, exhibiting high repeatability of our Hi-C experiments and analysis. These data suggested that the depletion of HNRNPU remodels long-range interactions at the compartment level.

We next examined compartment switching with Hnrnpu knockdown. The numbers of switched bins (200 kb each) in two independent knockdown experiments are 1094 (8.5\%) and 1099 (8.6\%), but those between two controls and two knockdown samples are only $113(0.9 \%)$ and $182(1.4 \%)$, respectively (Supplemental Fig. S6A). The percentages of compartment switching upon HNRNPU depletion are significantly higher than those between replicates in the same condition (generalized linear model with a 

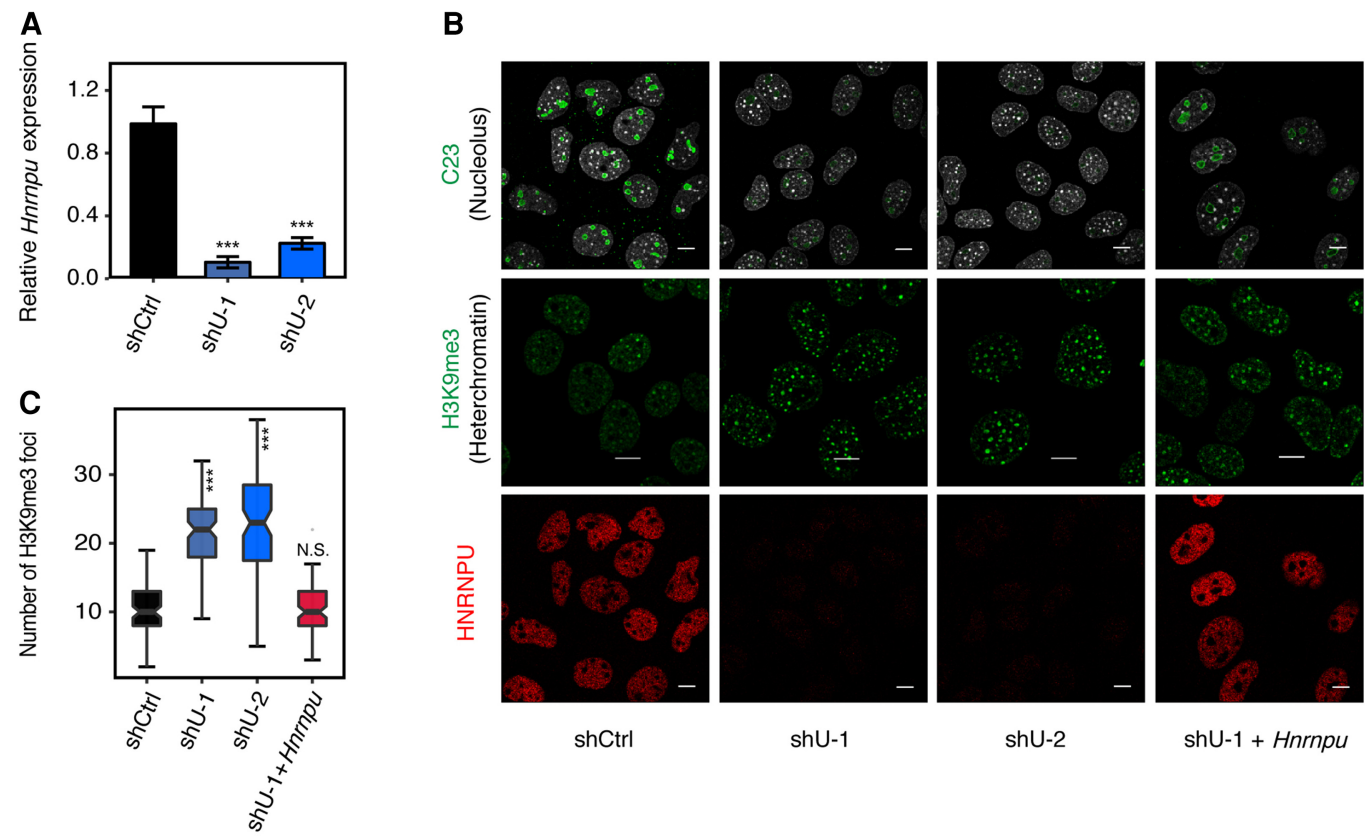

shCtrl

shU-1

shU-2

shU-1 + Hnrnpu

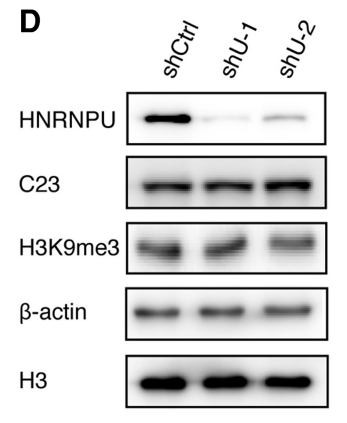

E
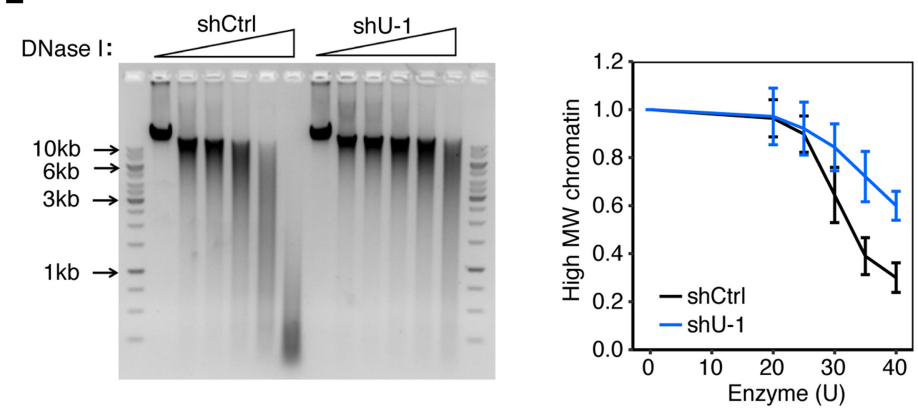

AML12 (Mouse)
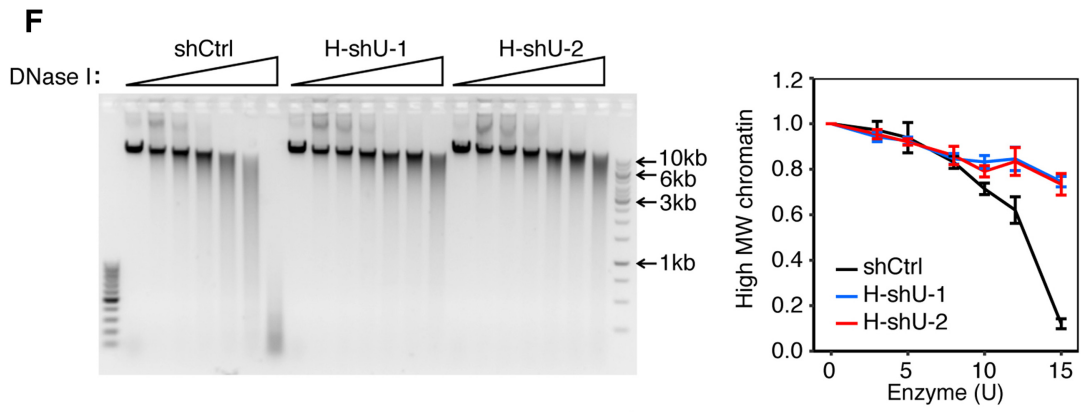

HepG2 (Human)

Figure 1. HNRNPU is required to maintain nuclear architecture. (A) Relative expression of Hnrnpu (against Actb) in the control (shCtrl) and Hnrnpu knockdown (shU-1 and shU-2) as detected by qRT-PCR. The error bars denote SD $(n=3)$. (***) $P<0.001, t$-test. (B) Immunofluorescence analyses of C23, H3K9me3, and HNRNPU. Scale bar, $10 \mu \mathrm{m}$. (C) Quantification of heterochromatin foci (H3K9me3) in response to Hnrnpu knockdown and rescuing. In total, 87 to 90 cells were analyzed for H3K9me3. $\left(^{* * *}\right) P<0.001$, Wilcoxon rank-sum test. $(D)$ Western blot analysis with antibodies against specified proteins; Actin and $\mathrm{H} 3$ as loading controls. $(E, F)$ Global chromatin structure as detected by DNase I digestion in AML12 (E) and HepG2 (F) cells. (Left) Gel images of genomic DNA digested by DNase I at different concentrations. (Right) Percentages of high molecular weight (MW) genomic DNA (>8 kb) determined by Image J software. Error bars indicate SD $(n=3)$.

binomial outcome, $P<2.2 \times 10^{-16}$ ). Additionally, switched compartments are highly consistent between the two replicates: A to B, $88 \%$ overlapping; B to A, $84 \%$ overlapping (Supplemental Fig. $\mathrm{S} 6 \mathrm{~B})$. By considering consistent results from replicates only, percentages of the switched region are $4.9 \%$ for A to B, and $2.6 \%$ for
B to A (Fig. 3E; Supplemental Table S3). There are 890 genes in compartments switched from A to B, and 747 genes in those shifted from $B$ to $A$. The gene density of these switched compartments is between those of unchanged A and B compartments (Supplemental Fig. S6C). 
A
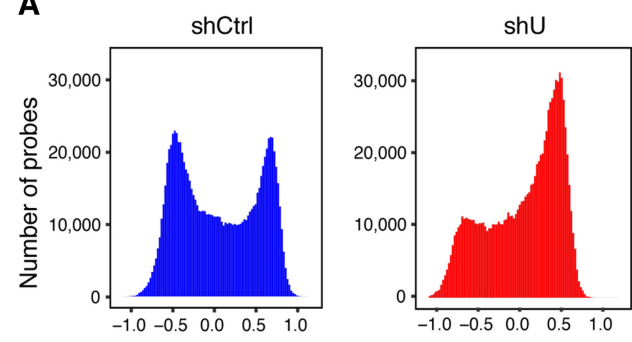

DamID smoothed ratio: $\log _{2}$ (Dam LB1 / Dam only)

C

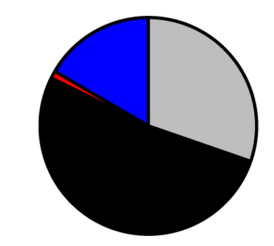

Gain of LADs $\quad 16.6 \%$ Loss of LADs $\quad 1.1 \%$

Stable LADs $\quad 52.0 \%$

Stable Non LADs $30.3 \%$
D

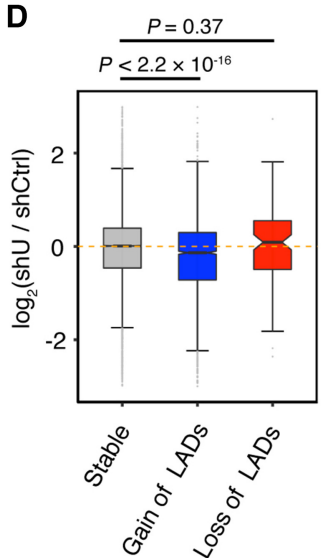

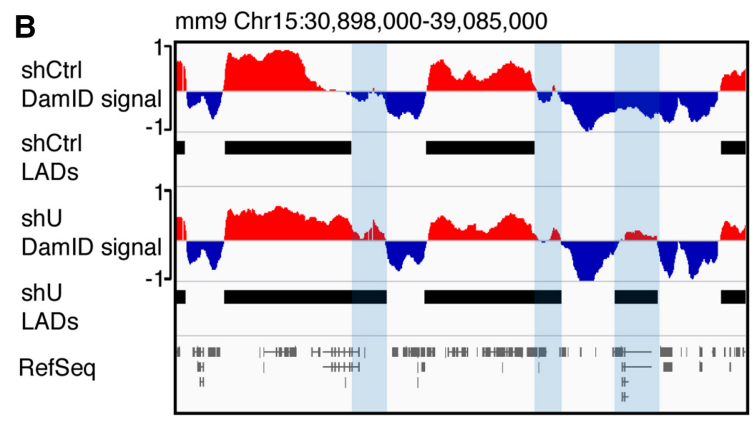

E

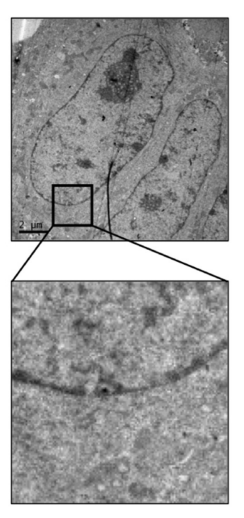

shCtrl

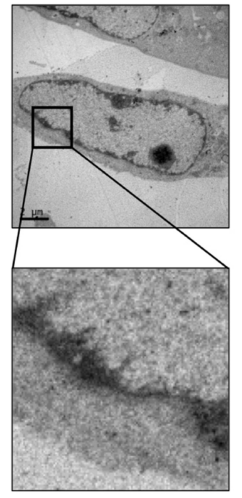

shU-1

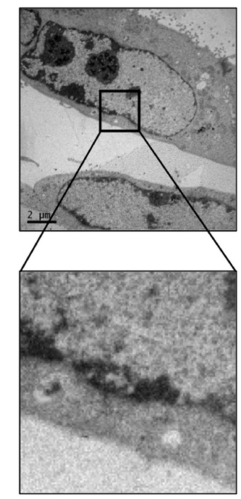

ShU-2

Figure 2. Depletion of HNRNPU increases lamina-chromatin association. ( $A$ ) Smoothed Lamin B1 DamID signals show different distributions in the control (shCtrl) and Hnrnpu knockdown (shU). (B) Lamin B1 (LB1) DamID signal tracks across one 8.2-Mb region on Chromosome 15. Black bars represent locations of LADs, and regions changed from non-LADs to LADs were highlighted in light blue. (C) Detailed changes of LADs with Hnrnpu knockdown. (D) Box plots showing gene expression changes in regions gained or lost of LADs. $P$-values were obtained by Wilcoxon rank-sum test. (E) Electron microscopy analysis of AML12 cells treated with different shRNAs. Scale bar, $2 \mu \mathrm{m}$.

Furthermore, the A-to-B compartment switching coincides with increases of chromatin-lamina interaction and decreases of gene expression, but B-to-A compartment switching displays opposite trends (Fig. 3F,G). The down-regulated genes paralleled with A-to-B switching are significantly enriched in GO terms related to liver functions, such as the $\mathrm{P} 450$ pathway and lipid transport (Supplemental Fig. S6D,E), suggesting a loss of cell identity resulted from changes of chromatin compartments.

\section{HNRNPU regulates TAD boundaries and interactions}

We next examined chromatin interactions at the TAD level. We calculated TAD boundaries using two replicates separately. The overlap between replicates in the same condition is higher than 95\% (Supplemental Fig. S7A,B). By considering only consistent data between replicates, we identified 2295 and 2263 TAD boundaries in the control and HNRNPU knockdown samples, respectively, and 2027 boundaries are shared between two conditions (Supplemental Fig. S7C; Supplemental Table S4). The median sizes of TADs are $920 \mathrm{~kb}$ in both conditions (Supplemental Fig. S7D). As visualized by the Hi-C contact map of a $10-\mathrm{Mb}$ region on mouse Chromosome 19, TAD patterns are largely invariant between the control and knockdown cells. However, finer chromatin contacts and insulation scores of TAD boundaries are changed (Fig. 4A).

To further investigate TAD interactions, we used $k$-means clustering to divide TAD boundaries into three categories: within compartment $\mathrm{A}$, within compartment $\mathrm{B}$, and between $\mathrm{A}$ and $\mathrm{B}$
(Fig. 4B). We then plotted insulation profiles centered by TAD boundaries and observed different patterns: (1) Within compartment A (or B), the knockdown is higher (or lower) than the control in the boundary and both sides; (2) between A and B, the knockdown is higher in the boundary and the A-side but lower in the B-side (Fig. 4C). Consistently, both intra- and inter-TAD interactions are significantly increased (or decreased) within A (or B) compartments, and inter-TAD interactions at the A/B boundaries are significantly increased (Fig. 4D).

Moreover, strengths are significantly decreased in TAD boundaries between compartment $\mathrm{A}$ and $\mathrm{B}\left(P<2.2 \times 10^{-16}\right.$, ANOVA) and in those within B compartments $\left(P=1.0 \times 10^{-5}\right)$ (Supplemental Fig. S7E), and the most substantial decreases of intensity are detected at the A/B boundaries (Fig. 4E). Consistently, $930(45.9 \%)$ of TAD boundaries decrease upon HNRNPU depletion, and $622(67 \%)$ of them are located at the edges of A and B compartments (Fig. 4F).

Thus, our data indicate that HNRNPU regulates TAD boundaries and TAD interactions in a compartment-specific manner, and the TAD boundaries at the borders of $\mathrm{A}$ and $\mathrm{B}$ compartments display the most considerable changes upon HNRNPU depletion.

\section{HNRNPU regulates chromatin loops}

To reveal roles of HNRNPU in 3D chromatin organization at finer scales, we investigated changes of chromatin loops. We first merged all contacts from control and knockdown samples for a 
Fan et al.
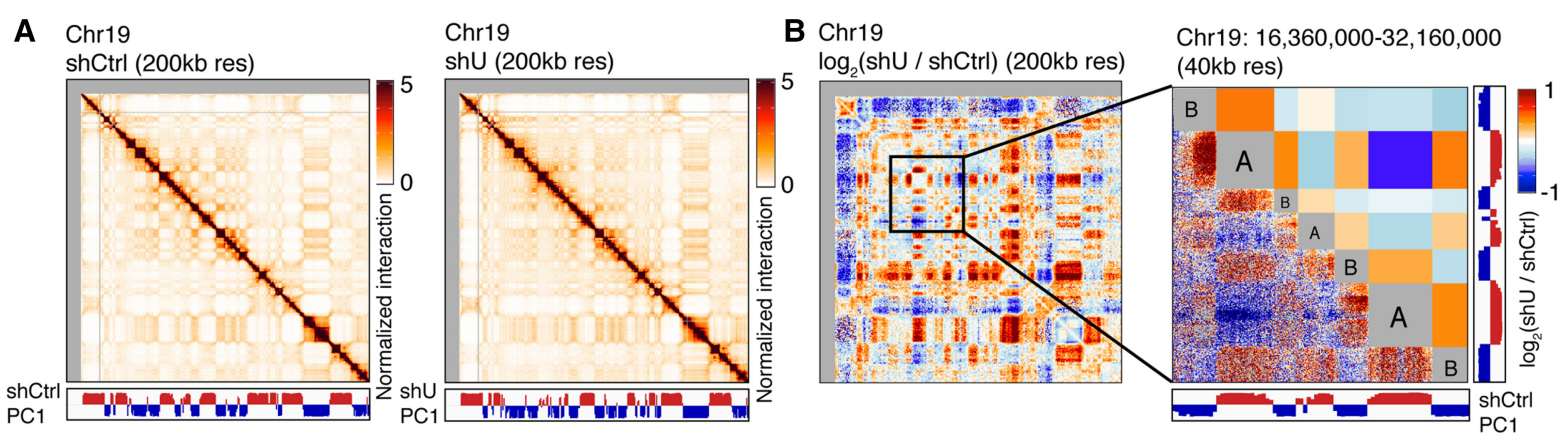

C
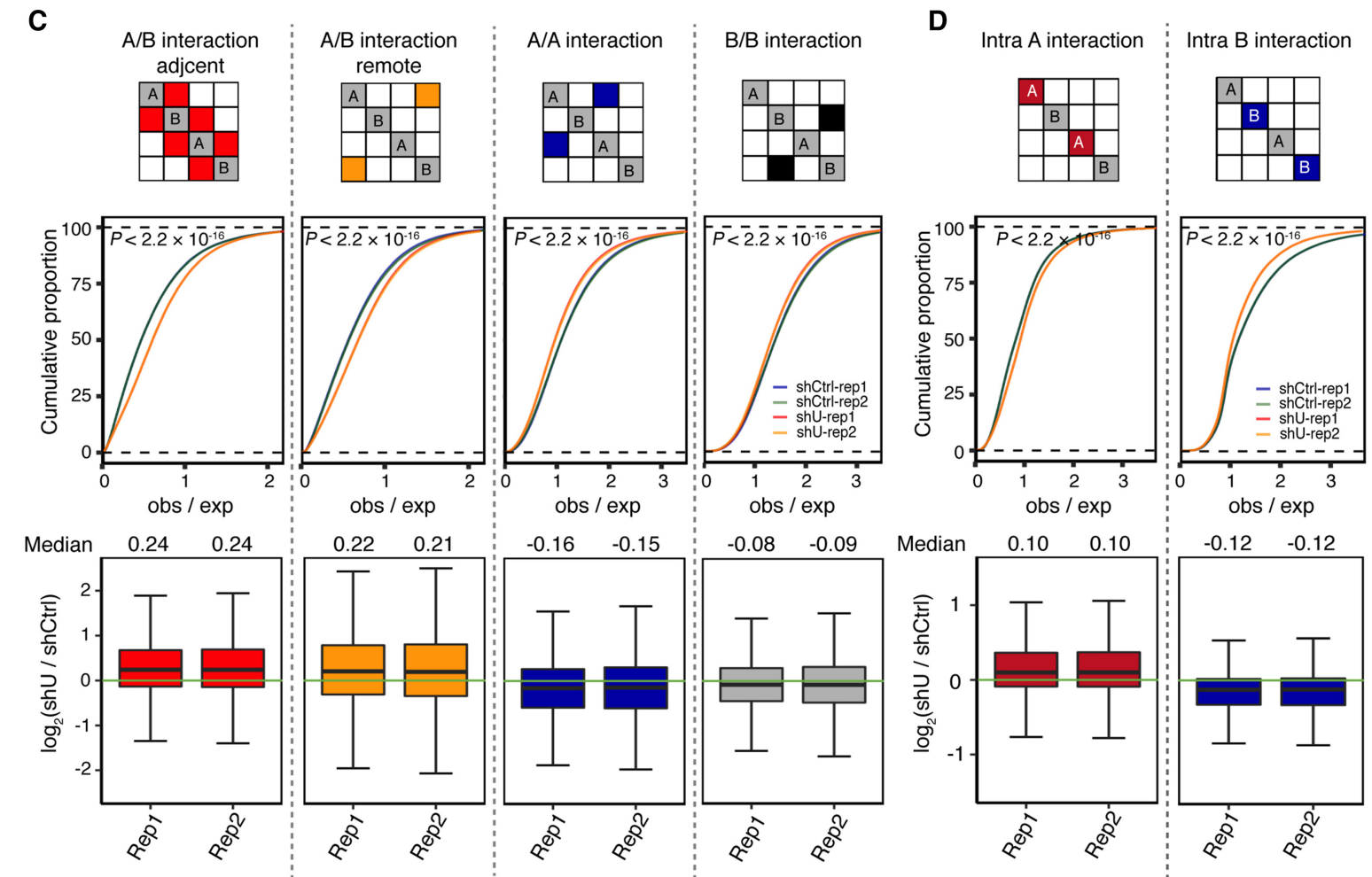

E
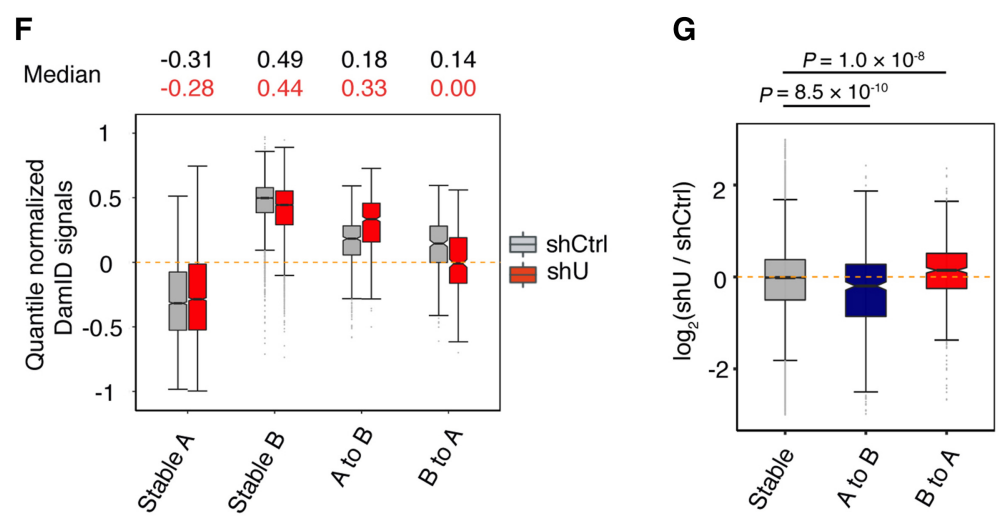

Figure 3. HNRNPU regulates chromatin compartment organization. (A) Hi-C contact maps of Chromosome 19 in (left) shCtrl- and (right) shU-treated cells at 200-kb resolution. Compartments A (red) and B (blue) are shown by PC1 eigenvectors. (B) (Left) $\log _{2}$ (shU/shCtrl) of Chromosome 19 binned at 200-kb resolution. (Right) $\log _{2}$ (shU/shCtrl) of $40-\mathrm{kb}$ bins (lower left) and averaged by compartments (upper right) in a 15.9-Mb region. (C,D) Statistics of interaction changes between $(C)$ and within $(D)$ compartments. (Top) Schematic view of regions examined. (Middle) Cumulative distribution of obs/exp values of interactions between compartments. P-values were obtained by two-factor ANOVA. (Bottom) Box plots showing the pairwise fold changes of interactions. Results from two replicates are presented. (E) Percentages of compartment switching between shCtrl- and shU-treated cells. $(F)$ The relationships between compartment switching and the lamina association (based on lamin B1 DamID data). (G) Box plots showing expression changes with compartment switching. $P$-values were obtained by Wilcoxon rank-sum test. 
A

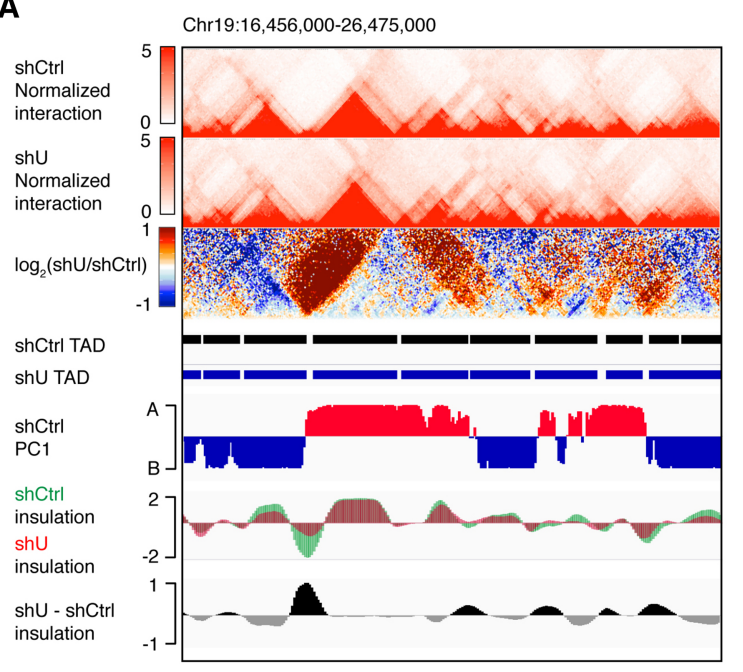

C
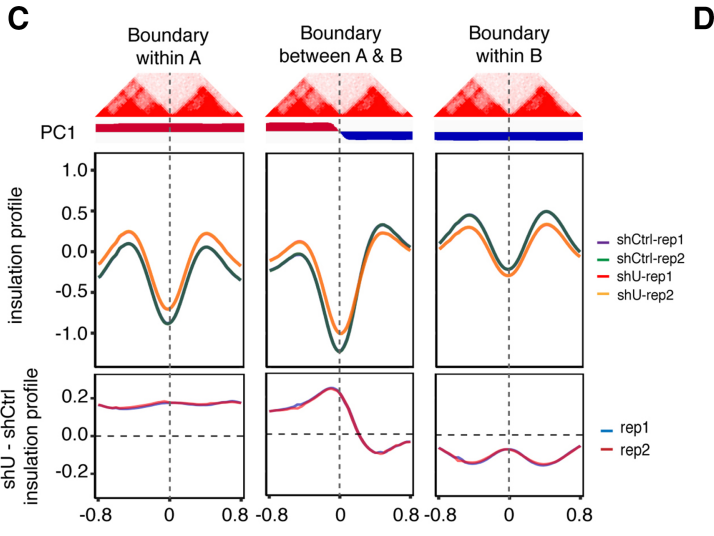

E
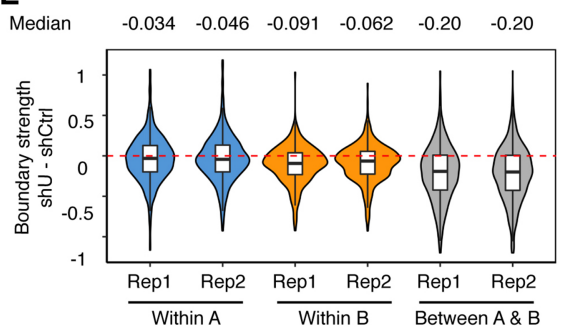

$\mathbf{F}$
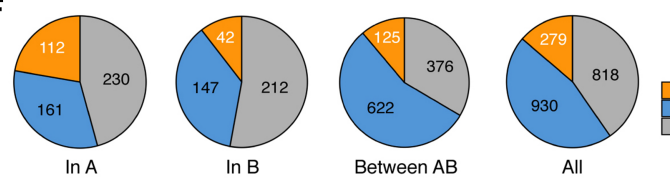

Increased

Decreased

Between $A B \quad$ All

Figure 4. HNRNPU regulates TAD interactions. $(A)$ A representative region showing interaction frequencies, insulation, and TAD boundaries at $40-\mathrm{kb}$ resolution. (B) $K$-means clustering of TAD boundaries with surrounding PC1 values. (C) Insulation profile around TAD boundaries. (Top) Schematic view of three classes of boundaries relative to compartments. (Middle) Medians of insulation profile for each sample. (Bottom) Differences of insulation profile for each category. (D) Quantification of inter- and intra-TAD interactions in compartment A, B, and between A and B. P-values were obtained by two-factor ANOVA. $(E)$ Violin plots showing degrees of boundary strength changes. Results from two replicates are presented. $(F)$ Percentage of changed TAD boundaries in different categories.

more accurate loop detection and identified, in total, 7994 chromatin loops (Supplemental Table S5). The overall strengths of chromatin loops are reduced in the HNRNPU-depleted cells as

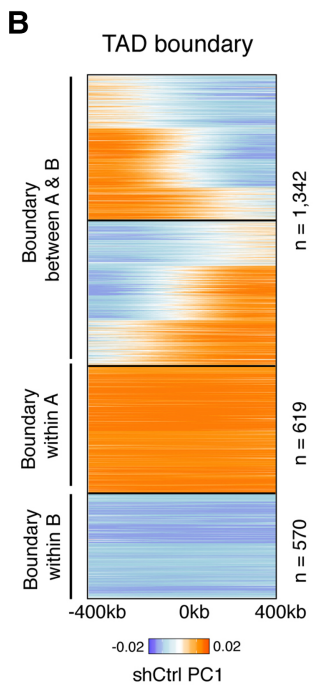

shown by the aggregate signal from all loops (Fig. 5A). To test the statistical significance of loop changes upon HNRNPU depletion, we compared the intensity of loops (peaks) and "fold changes" of peaks relative to four neighboring regions (Fig. 5B), as proposed previously (Rao et al. 2014). In the HNRNPU-depleted cells, the intensity of peaks (Fig. 5C) and their relative ratios to adjacent regions (Fig. 5D) are all significantly reduced. Then, we defined differential loops only if all the five values (peak intensity and ratios relative to four neighboring regions) change in the same direction. In total, we identified 4651 $(58.2 \%)$ decreased and $680(8.5 \%)$ increased loops upon HNRNPU knockdown (Fig. 5E). Two representative regions showing decreased chromatin loops are provided in Figure 5F.

\section{HNRNPU associates with CTCF and RAD21}

Next, we explored how HNRNPU affects chromatin organization. High-throughput mass spectrometry data suggested that HNRNPU might interact with CTCF (Hutchins et al. 2010). Our immunofluorescence data indicated that both CTCF and RAD21, a component of the cohesin complex, are partially colocalized with HNRNPU in the nucleus (Fig. 6A). Co-immunoprecipitation experiments further validated that HNRNPU, CTCF, and RAD21 could be associated (Fig. 6B).

Then, we mapped genome-wide chromatin binding of CTCF, RAD21, and HNRNPU in AML12 cells (Fig. 6C; Supplemental Tables S6, S7). For CTCF and RAD21, we used regular ChIP-seq; for HNRNPU, we applied bioChIP-seq, which is based on in vivo biotinylation of interested proteins by the BirA enzyme (He and $\mathrm{Pu}$ 2010). Our HNRNPU bioChIP-seq experiments were carefully controlled by examining expression and localization of tagged HNRNPU proteins (Supplemental Fig. S8). By comparing with the BirA-only control, we identified 5285 HNRNPU peaks. Strikingly, more than $80 \%$ of HNRNPU peaks overlap with CTCF or RAD21, and $59.6 \%$ of HNRNPU peaks overlap with both (Fig. $6 \mathrm{D})$, further supporting the interaction among these three proteins. However, we still cannot determine whether the interaction is direct or indirect.

Furthermore, HNRNPU peaks are enriched at TAD boundaries (Supplemental Fig. S9A), and intensities of HNRNPU-associated boundaries are significantly higher than those not associated 

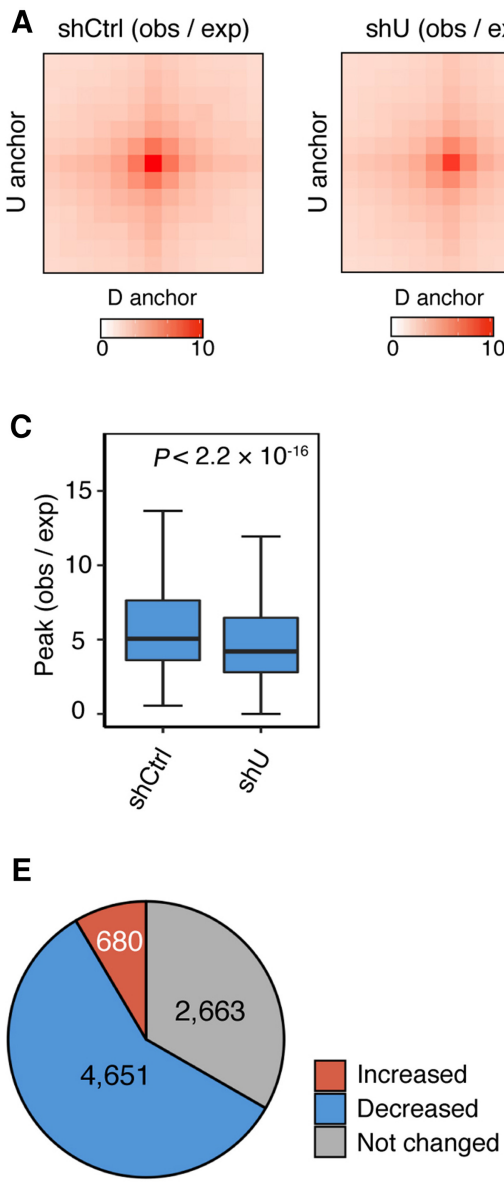
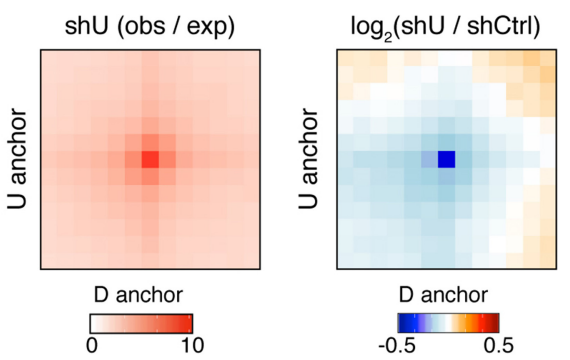
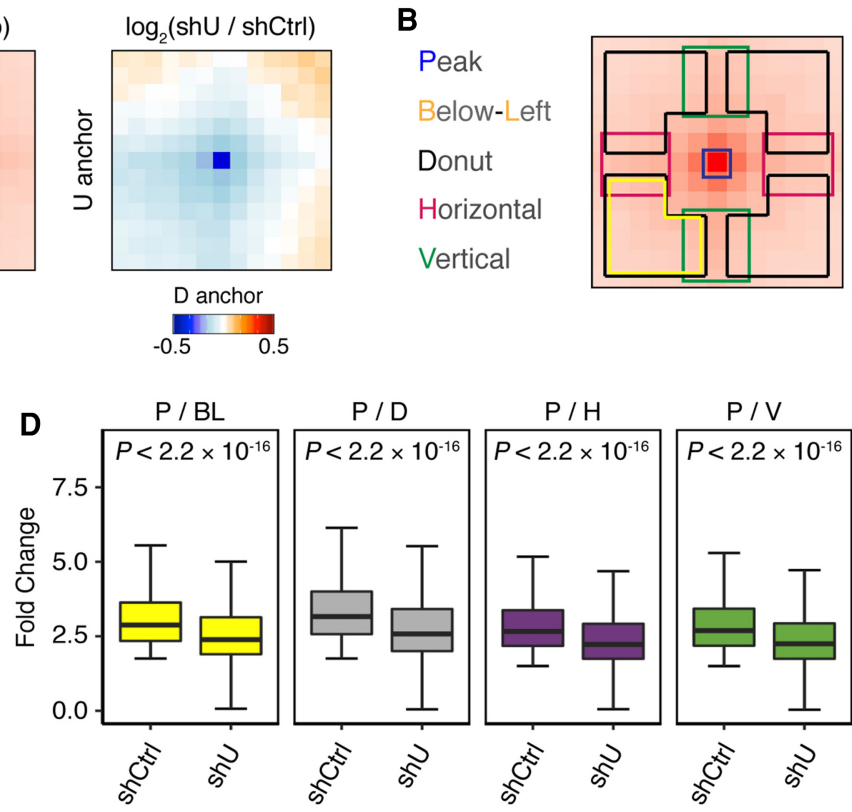

$\mathbf{F}$
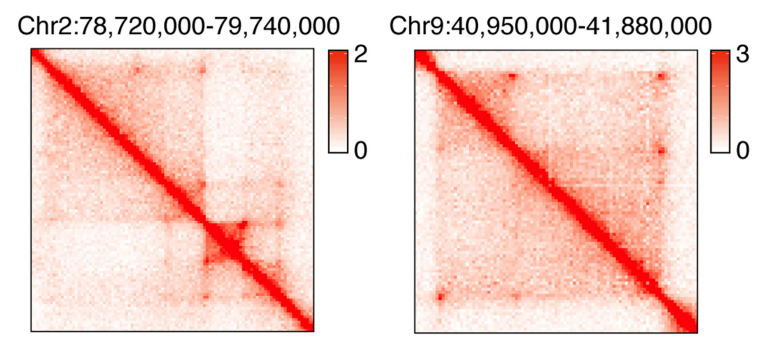

Upper tri: shCtrl (Normalized interaction)

Lower tri: shU (Normalized interaction)

Figure 5. The intensity of chromatin loops decreases upon HNRNPU depletion. $(A)$ Mean aggregate signals of 7994 loops by summing submatrices surrounding each peak (10 kb $\times 10 \mathrm{~kb}$ for each pixel). (B) Schematic view of loop center (peak) and adjacent areas: below-left (yellow), donut (black), horizontal (purple), and vertical (green). (C) Box plots showing the intensity of peaks in shCtrl- and shU-treated cells. $P$-values were obtained by Wilcoxon ranksum test. $(D)$ Box plots showing fold change of peak signal relative to adjacent areas. $P$-values were obtained by Wilcoxon rank-sum test. ( $E$ ) Percentage of changed chromatin loops upon HNRNPU depletion. $(F)$ Representative regions showing decreases of chromatin loops.

with HNRNPU (Supplemental Fig. S9B). Interestingly, upon HNRNPU depletion, only boundaries bound by CTCF and RAD21 are significantly decreased in their strengths (Supplemental Fig. S9C).

To test whether HNRNPU affects the binding of CTCF and RAD21 on chromatin, we compared ChIP-seq data from control and knockdown cells (Supplemental Fig. S10A,B). While CTCF binding is almost unchanged, $3816(9.8 \%)$ RAD21 peaks are significantly reduced in their binding strengths (Supplemental Fig. S10C,D). These decreased RAD21 peaks are highly associated with CTCF binding (Supplemental Fig. S10E). Concordantly, chromatin loops associated with decreased RAD21 binding are significantly weaker than those associated with invariant RAD21 peaks (Supplemental Fig. S10F,G), but proteins levels of CTCF and RAD21 remain unchanged upon HNRNPU depletion (Supplemental Fig. S10H).

\section{HNRNPU associates with active chromatin}

Finally, we examined the distribution and strength of HNRNPU binding relative to genes. Surprisingly, $60 \%$ of HNRNPU peaks lo- cate at promoters (Fig. 6E). Further, binding strengths of HNRNPU near transcription start sites (TSSs) are positively correlated with levels of gene expression (Fig. 6F). Consistently, 90\% of HNRNPU peaks are within compartment A or non-LADs (Fig. 6G). Therefore, our data indicated that HNRNPU mainly associates with active chromatin.

\section{Discussion}

In conclusion, using mouse hepatocytes as the model, we demonstrate that HNRNPU functions as a major regulator of the 3D genome, regulating multiple levels of chromatin architectures, including LADs, compartments, TADs, and chromatin loops, partially through cooperating with CTCF and RAD21.

Although the concept of the NM has been disputed for decades (Pederson 2000; Nickerson 2001), the NM is still being hypothesized as a platform for 3D genome organization (Razin et al. 2014). Besides the HNRNPU data presented here, topoisomerase II beta, another NM-associated protein, also associates with cohesin and CTCF at TAD boundaries (Uuskula-Reimand et al.

\section{Genome Research}

www.genome.org 
A
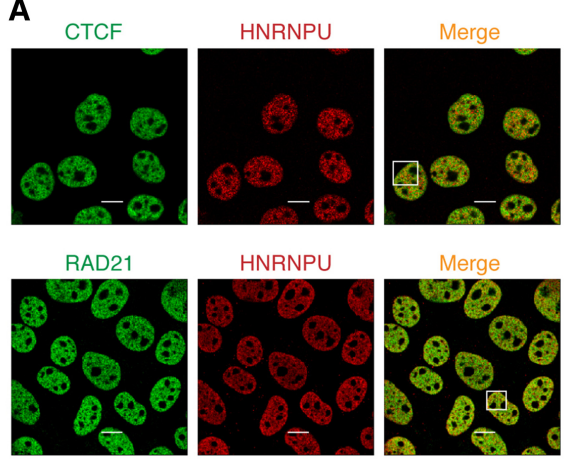

C

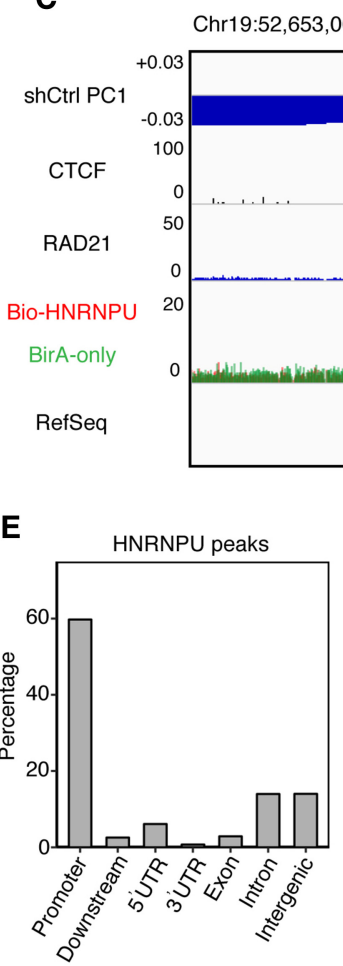

B

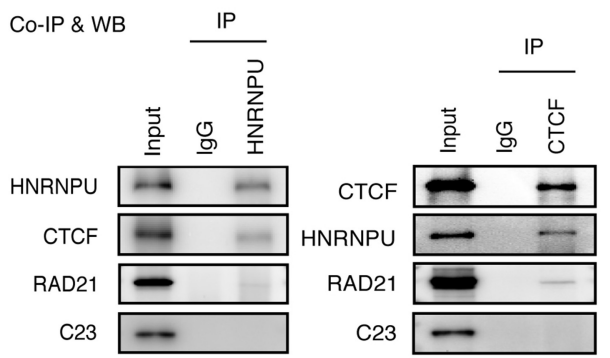

D

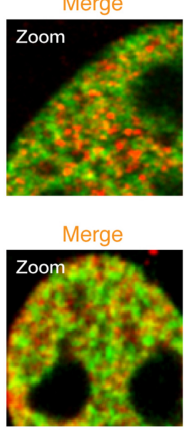

CTCF peak

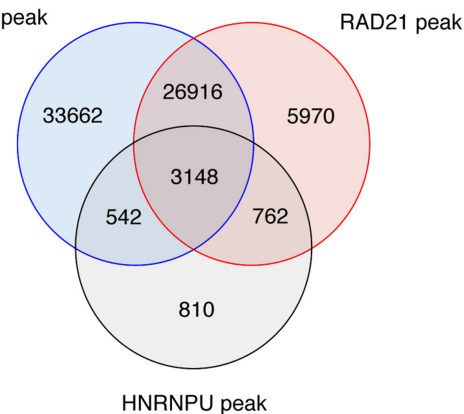

$\mathbf{F}$

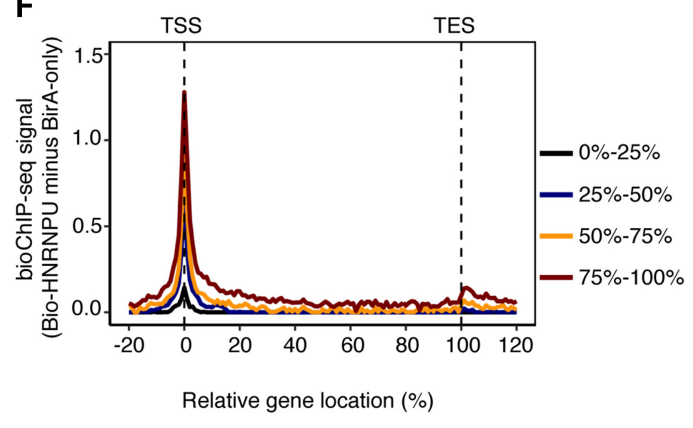

G

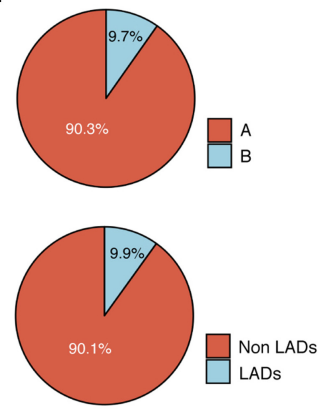

Figure 6. HNRNPU associates with CTCF and RAD21. $(A)$ Immunofluorescent staining displays partial colocalization of HNRNPU (red) and CTCF or RAD21 (green) in AML12 cells. Scale bar, $10 \mu \mathrm{m}$. (B) Endogenous co-immunoprecipitation with HNRNPU (left) or CTCF (right) antibodies, and blots with indicated antibodies. (C) ChIP-seq tracks of CTCF and RAD21; bioChIP-seq tracks of HNRNPU and BirA only (negative control) on a 1.3-Mb region on Chromosome 19. (D) Venn diagram showing overlaps between peaks of CTCF, RAD21, and HNRNPU. (E) Distribution of HNRNPU peaks relative to genes. $(F)$ Average profiles of HNRNPU bioChIP-seq signal of genes at different expression levels (100\% denotes the highest expression). ( $G$ ) Distribution of HNRNPU peaks relative to compartments and LADs.

2016), suggesting general roles of the NM and/or its associated proteins in $3 \mathrm{D}$ genome organization. As a potential mediator connecting chromatin and NM, HNRNPU mainly associates with A compartments, as indicated in Figure 6G. HNRNPU may maintain proper chromatin architecture by "stabilizing" chromatin to the inner NM. The depletion of HNRNPU may decrease the constraint on chromatin, therefore remodeling chromatin-chromatin and chromatin-lamina interactions (Supplemental Fig. S11).

During the review process of our manuscript, Nick Gilbert and colleagues reported that, primarily based on FISH, biochemistry, and mutagenesis, HNRNPU/SAF-A is involved in de-compaction of chromatin via oligomerization with chromatin-associated RNAs (caRNAs), mainly in gene-rich regions (Nozawa et al.
2017). In the current study, we showed that HNRNPU depletion leads to global chromatin condensation, an increase of LADs from $53.1 \%$ to $68.6 \%$ of the genome, compartment switching on $7.5 \%$ of the genome, and a significant decrease of TAD boundary strengths and chromatin loop intensities. Therefore, these two studies are highly complementary, demonstrating important roles of HNRNPU in the higher-order chromatin organization.

Furthermore, the Gilbert study demonstrated that the regulatory role of HNRNPU on chromatin depends on transcription and caRNAs (Nozawa et al. 2017). Our study indicated that HNRNPU strongly associates with active genes and with CTCFand RAD21-bound regions. These observations suggested that caRNAs could be involved in the organization of higher-order 
chromatin via cooperating with RNA binding proteins (such as HNRNPU), structural proteins (such as CTCF and cohesin), and the transcription machinery, presumably.

Additionally, as a protein binding most kinds of RNAs (Xiao et al. 2012), HNRNPU also interacts with long noncoding RNAs (lncRNAs) such as Xist (Chu et al. 2015; McHugh et al. 2015) and Firre (Hacisuleyman et al. 2014), both of which are known regulators of chromatin interactions at specific regions. Other IncRNAs, including LincGET and Blen1, are also found to be interacting with HNRNPU (Wang et al. 2016a; Mi et al. 2017). These studies suggest potential lncRNA-dependent roles of HNRNPU in genome organization of specific loci, which awaits further investigation.

Our data indicated that HNRNPU affects the strengths of more than $40 \%$ of TAD boundaries. Additionally, TAD boundaries are enriched for the binding of HNRNPU and other known 3D genome regulators including CTCF, RAD21, and SMARCA4 (Dixon et al. 2012; Sofueva et al. 2013; Barutcu et al. 2016b). Our study further indicated that HNRNPU proteins interact with CTCF and RAD21. Thus, these "boundary factors" may form macrocomplexes, directly or indirectly, to segregate TADs. Further studies are needed to identify components and functional roles of these potential macrocomplexes and to reveal their dynamics and dysfunction in development and diseases.

The HNRNPU-deficient mice displayed phenotypes of postimplantation lethality (Roshon and Ruley 2005) and impaired heart development as demonstrated by conditional knockout (Ye et al. 2015). Human de novo mutations or microdeletions of the Hnrnpu gene are associated with brain disorders (Epi4K Consortium et al. 2013; Gupta et al. 2014). The experimental observations in both humans and mice suggest that HNRNPU could regulate genome organization in vivo in development and diseases.

\section{Methods}

\section{RNAi and rescue}

For RNAi experiments, shRNA plasmids were constructed by cloning the target sequences to the pLKO.1-puro vector: AGA TCATGGCCGAGGGTATTT (mouse-shU-1), TGCCCGTAAGAA GCGAAATTT (mouse-shU-2), AGATCATGGCCGTGGATATTT (human-shU-1), GACTCTGTACTGCTCATATTA (human-shU-2), and ACTCGACACTATAGTATCTCA (negative control). For rescue experiments, mouse Hnrnpu cDNA was mutated for the targets of shU-1. AML12 cells were treated with shRNAs or expression vectors by lentivirus-based systems for $5 \mathrm{~d}$.

\section{Western blotting}

The cell lysates were blotted against primary antibodies as follows: anti- $\beta$ actin (AOGMA, AGM11086), anti-H3 (Abcam, ab1791), anti-H3K9me3 (ABclonal, A2360), anti-C23 (Proteintech, 105561-AP), anti-HNRNPU (Abcam, ab20666), and anti-RAD21 (Abcam, ab992). The blots were visualized with peroxidase-coupled secondary antibodies.

\section{Immunofluorescence}

Immunofluorescence was conducted with the same antibodies used for Western blots except for HNRNPU (Abcam, ab10297). The slides were imaged with a confocal laser scanning microscopy (Leica, SP5).

\section{Electron microscopy imaging}

Cells were fixed with $2.5 \%$ glutaraldehyde and further processed and imaged by the EM facility at the Shanghai Institute of Biochemistry and Cell Biology (SIBCB).

\section{DamID}

DamID experiments were performed as described (Guelen et al. 2008). Two biological replicates were conducted for the control and Hnrnpu knockdown AML-12 cells. Microarray data were analyzed as previously described (Fu et al. 2015).

\section{In situ Hi-C}

The in situ Hi-C libraries were prepared as previously described (Rao et al. 2014). Two biological replicates were performed for both control and HNRNPU-depleted AML12 cells. The libraries were then sequenced via the Illumina HiSeq $X$ Ten system. Detailed information about Hi-C data analysis is provided in the Supplemental Methods.

\section{ChIP-seq and bioChIP-seq}

ChIP experiments were performed as previously described (Wen et al. 2008) with antibodies against CTCF or RAD21. For HNRNPU, we applied bioChIP experiments as previously described (He and Pu 2010; Roux et al. 2012) with some modifications. Detailed information of bioChIP experiments and ChIPseq data analysis are provided in the Supplemental Methods.

\section{Data access}

The raw and processed DamID, RNA-seq, ChIP-seq, and Hi-C data sets from this study have been submitted to the NCBI Gene Expression Omnibus (GEO; http://www.ncbi.nlm.nih.gov/geo/) under accession number GSE95116.

\section{Acknowledgments}

We thank members of the Wen lab for their support and suggestions, X. Zhao for assistance with ChIP-seq, and W. Qiu for assistance with Hi-C library preparation. This study was supported by the National Basic Research Program of China (2015CB943000 to B.W.) and the National Natural Science Foundation of China (31371296 to B.W.).

Author contributions: H.F. and B.W. conceived and designed this study. H.F. performed most of the experiments except for DamID assay (by X.H.), Hi-C library preparation (Q.W.), Co-IP (J.W.), and DamID microarray experiments (L.Z. and F.Z.). P.L. analyzed the data, with the guidance of H.W., Y.L., and X.Z. L.C. and Q.-Q.T. provided some experimental support. B.W., H.F., and P.L. prepared the manuscript with input from all authors.

\section{References}

Bailey SD, Zhang X, Desai K, Aid M, Corradin O, Cowper-Sal Lari R, AkhtarZaidi B, Scacheri PC, Haibe-Kains B, Lupien M. 2015. ZNF143 provides sequence specificity to secure chromatin interactions at gene promoters. Nat Commun 2: 6186.

Barutcu AR, Hong D, Lajoie BR, McCord RP, van Wijnen AJ, Lian JB, Stein JL, Dekker J, Imbalzano AN, Stein GS. 2016a. RUNX1 contributes to higherorder chromatin organization and gene regulation in breast cancer cells. Biochim Biophys Acta 1859: 1389-1397.

Barutcu AR, Lajoie BR, Fritz AJ, McCord RP, Nickerson JA, van Wijnen AJ, Lian JB, Stein JL, Dekker J, Stein GS, et al. 2016b. SMARCA4 regulates gene expression and higher-order chromatin structure in proliferating mammary epithelial cells. Genome Res 26: 1188-1201.

\section{Genome Research}

www.genome.org 
Bickmore WA. 2013. The spatial organization of the human genome. Annu Rev Genomics Hum Genet 14: 67-84.

Chu C, Zhang QC, da Rocha ST, Flynn RA, Bharadwaj M, Calabrese JM, Magnuson T, Heard E, Chang HY. 2015. Systematic discovery of Xist RNA binding proteins. Cell 161: 404-416.

Cremer T, Cremer M. 2010. Chromosome territories. Cold Spring Harb Perspect Biol 2: a003889.

Dekker J, Mirny L. 2016. The 3D genome as moderator of chromosomal communication. Cell 164: 1110-1121.

Dekker J, Misteli T. 2015. Long-range chromatin interactions. Cold Spring Harb Perspect Biol 7: a019356.

Dixon JR, Selvaraj S, Yue F, Kim A, Li Y, Shen Y, Hu M, Liu JS, Ren B. 2012. Topological domains in mammalian genomes identified by analysis of chromatin interactions. Nature 485: 376-380.

Dixon JR, Jung I, Selvaraj S, Shen Y, Antosiewicz-Bourget JE, Lee AY, Ye Z, Kim A, Rajagopal N, Xie W, et al. 2015. Chromatin architecture reorganization during stem cell differentiation. Nature 518: 331-336.

Dixon JR, Gorkin DU, Ren B. 2016. Chromatin domains: the unit of chromosome organization. Mol Cell 62: 668-680.

Epi4K Consortium, Epilepsy Phenome/Genome Project, Allen AS, Berkovic SF, Cossette P, Delanty N, Dlugos D, Eichler EE, Epstein MP, Glauser T, et al. 2013. De novo mutations in epileptic encephalopathies. Nature 501: $217-221$.

Fackelmayer FO, Dahm K, Renz A, Ramsperger U, Richter A. 1994. Nucleicacid-binding properties of hnRNP-U/SAF-A, a nuclear-matrix protein which binds DNA and RNA in vivo and in vitro. Eur J Biochem 221: 749-757.

Flavahan WA, Drier Y, Liau BB, Gillespie SM, Venteicher AS, StemmerRachamimov AO, Suva ML, Bernstein BE. 2016. Insulator dysfunction and oncogene activation in IDH mutant gliomas. Nature 529: 110-114.

Franke M, Ibrahim DM, Andrey G, Schwarzer W, Heinrich V, Schopflin R, Kraft K, Kempfer R, Jerkovic I, Chan WL, et al. 2016. Formation of new chromatin domains determines pathogenicity of genomic duplications. Nature 538: 265-269.

Fu Y, Lv P, Yan G, Fan H, Cheng L, Zhang F, Dang Y, Wu H, Wen B. 2015. MacroH2A1 associates with nuclear lamina and maintains chromatin architecture in mouse liver cells. Sci Rep 5: 17186.

Fudenberg G, Imakaev M, Lu C, Goloborodko A, Abdennur N, Mirny LA. 2016. Formation of chromosomal domains by loop extrusion. Cell Rep 15: $2038-2049$.

Getzenberg RH, Pienta KJ, Ward WS, Coffey DS. 1991. Nuclear structure and the three-dimensional organization of DNA. J Cell Biochem 47: 289-299.

Ghirlando R, Felsenfeld G. 2016. CTCF: making the right connections. Genes Dev 30: 881-891.

Gohring F, Fackelmayer FO. 1997. The scaffold/matrix attachment region binding protein hnRNP-U (SAF-A) is directly bound to chromosomal DNA in vivo: a chemical cross-linking study. Biochemistry 36: 8276-8283.

Gomez-Diaz E, Corces VG. 2014. Architectural proteins: regulators of 3D genome organization in cell fate. Trends Cell Biol 24: 703-711.

Groschel S, Sanders MA, Hoogenboezem R, de Wit E, Bouwman BA, Erpelinck C, van der Velden VH, Havermans M, Avellino R, van Lom $\mathrm{K}$, et al. 2014. A single oncogenic enhancer rearrangement causes concomitant EVI1 and GATA2 deregulation in leukemia. Cell 157: 369-381.

Guelen L, Pagie L, Brasset E, Meuleman W, Faza MB, Talhout W, Eussen BH, de Klein A, Wessels L, de Laat W, et al. 2008. Domain organization of human chromosomes revealed by mapping of nuclear lamina interactions. Nature 453: 948-951.

Guo Y, Xu Q, Canzio D, Shou J, Li J, Gorkin DU, Jung I, Wu H, Zhai Y, Tang Y, et al. 2015. CRISPR inversion of CTCF sites alters genome topology and enhancer/promoter function. Cell 162: 900-910.

Gupta R, Agarwal M, Boqqula VR, Phadke RV, Phadke SR. 2014. Hemiconvulsion-hemiplegia-epilepsy syndrome with 1q44 microdeletion: causal or chance association. Am J Med Genet A 164A: 186-189.

Hacisuleyman E, Goff LA, Trapnell C, Williams A, Henao-Mejia J, Sun L, McClanahan P, Hendrickson DG, Sauvageau M, Kelley DR, et al. 2014 Topological organization of multichromosomal regions by the long intergenic noncoding RNA Firre. Nat Struct Mol Biol 21: 198-206.

Hasegawa Y, Brockdorff N, Kawano S, Tsutui K, Tsutui K, Nakagawa S. 2010. The matrix protein hnRNP $\mathrm{U}$ is required for chromosomal localization of Xist RNA. Dev Cell 19: 469-476.

He A, Pu WT. 2010. Genome-wide location analysis by pull down of in vivo biotinylated transcription factors. Curr Protoc Mol Biol Chapter 21: Unit 2120.

Heidari N, Phanstiel DH, He C, Grubert F, Jahanbani F, Kasowski M, Zhang MQ, Snyder MP. 2014. Genome-wide map of regulatory interactions in the human genome. Genome Res 24: 1905-1917.

Hnisz D, Day DS, Young RA. 2016. Insulated neighborhoods: structural and functional units of mammalian gene control. Cell 167: 1188-1200.
Hu J, Zhang Y, Zhao L, Frock RL, Du Z, Meyers RM, Meng FL, Schatz DG, Alt FW. 2015. Chromosomal loop domains direct the recombination of antigen receptor genes. Cell 163: 947-959.

Hutchins JR, Toyoda Y, Hegemann B, Poser I, Heriche JK, Sykora MM, Augsburg M, Hudecz O, Buschhorn BA, Bulkescher J, et al. 2010. Systematic analysis of human protein complexes identifies chromosome segregation proteins. Science 328: 593-599.

Lieberman-Aiden E, van Berkum NL, Williams L, Imakaev M, Ragoczy T, Telling A, Amit I, Lajoie BR, Sabo PJ, Dorschner MO, et al. 2009. Comprehensive mapping of long-range interactions reveals folding principles of the human genome. Science 326: 289-293.

Linnemann AK, Krawetz SA. 2009. Maintenance of a functional higher order chromatin structure: the role of the nuclear matrix in normal and disease states. Gene Ther Mol Biol 13: 231-243.

Lucas JS, Zhang Y, Dudko OK, Murre C. 2014. 3D trajectories adopted by coding and regulatory DNA elements: first-passage times for genomic interactions. Cell 158: 339-352.

Lupianez DG, Kraft K, Heinrich V, Krawitz P, Brancati F, Klopocki E, Horn D, Kayserili H, Opitz JM, Laxova R, et al. 2015. Disruptions of topological chromatin domains cause pathogenic rewiring of gene-enhancer interactions. Cell 161: 1012-1025.

McDonald OG, Wu H, Timp W, Doi A, Feinberg AP. 2011. Genome-scale epigenetic reprogramming during epithelial-to-mesenchymal transition. Nat Struct Mol Biol 18: 867-874.

McHugh CA, Chen CK, Chow A, Surka CF, Tran C, McDonel P, PandyaJones A, Blanco M, Burghard C, Moradian A, et al. 2015. The Xist IncRNA interacts directly with SHARP to silence transcription through HDAC3. Nature 521: 232-236.

Mi L, Zhao XY, Li S, Yang G, Lin JD. 2017. Conserved function of the long noncoding RNA Blnc1 in brown adipocyte differentiation. Mol Metab 6: 101-110.

Narendra V, Rocha PP, An D, Raviram R, Skok JA, Mazzoni EO, Reinberg D. 2015. CTCF establishes discrete functional chromatin domains at the Hox clusters during differentiation. Science 347: 1017-1021.

Nickerson J. 2001. Experimental observations of a nuclear matrix. J Cell Sci 114: $463-474$

Nora EP, Lajoie BR, Schulz EG, Giorgetti L, Okamoto I, Servant N, Piolot T, van Berkum NL, Meisig J, Sedat J, et al. 2012. Spatial partitioning of the regulatory landscape of the X-inactivation centre. Nature 485: 381-385.

Nozawa RS, Boteva L, Soares DC, Naughton C, Dun AR, Buckle A, Ramsahoye B, Bruton PC, Saleeb RS, Arnedo M, et al. 2017. SAF-A regulates interphase chromosome structure through oligomerization with chromatin-associated RNAs. Cell 169: 1214-1227.e18.

Pederson T. 2000. Half a century of "the nuclear matrix". Mol Biol Cell 11: 799-805.

Pope BD, Ryba T, Dileep V, Yue F, Wu W, Denas O, Vera DL, Wang Y, Hansen RS, Canfield TK, et al. 2014. Topologically associating domains are stable units of replication-timing regulation. Nature 515: 402-405.

Rao SS, Huntley MH, Durand NC, Stamenova EK, Bochkov ID, Robinson JT, Sanborn AL, Machol I, Omer AD, Lander ES, et al. 2014. A 3D map of the human genome at kilobase resolution reveals principles of chromatin looping. Cell 159: 1665-1680.

Razin SV, Iarovaia OV, Vassetzky YS. 2014. A requiem to the nuclear matrix: from a controversial concept to $3 \mathrm{D}$ organization of the nucleus. Chromosoma 123: 217-224.

Roshon MJ, Ruley HE. 2005. Hypomorphic mutation in hnRNP U results in post-implantation lethality. Transgenic Res 14: 179-192.

Roux KJ, Kim DI, Raida M, Burke B. 2012. A promiscuous biotin ligase fusion protein identifies proximal and interacting proteins in mammalian cells. J Cell Biol 196: 801-810.

Rowley MJ, Corces VG. 2016. The three-dimensional genome: principles and roles of long-distance interactions. Curr Opin Cell Biol 40: 8-14.

Sanborn AL, Rao SS, Huang SC, Durand NC, Huntley MH, Jewett AI Bochkov ID, Chinnappan D, Cutkosky A, Li J, et al. 2015. Chromatin extrusion explains key features of loop and domain formation in wild-type and engineered genomes. Proc Natl Acad Sci 112: E6456-E6465.

Schmitt AD, Hu M, Jung I, Xu Z, Qiu Y, Tan CL, Li Y, Lin S, Lin Y, Barr CL, et al. 2016. A compendium of chromatin contact maps reveals spatially active regions in the human genome. Cell Rep 17: 2042-2059.

Seitan VC, Faure AJ, Zhan Y, McCord RP, Lajoie BR, Ing-Simmons E, Lenhard B, Giorgetti L, Heard E, Fisher AG, et al. 2013. Cohesin-based chromatin interactions enable regulated gene expression within preexisting architectural compartments. Genome Res 23: 2066-2077.

Sexton T, Yaffe E, Kenigsberg E, Bantignies F, Leblanc B, Hoichman M, Parrinello H, Tanay A, Cavalli G. 2012. Three-dimensional folding and functional organization principles of the Drosophila genome. Cell 148: $458-472$.

Sofueva S, Yaffe E, Chan WC, Georgopoulou D, Vietri Rudan M, MiraBontenbal H, Pollard SM, Schroth GP, Tanay A, Hadjur S. 2013. Cohesin-mediated interactions organize chromosomal domain architecture. EMBO J 32: 3119-3129. 
Fan et al.

Stevens TJ, Lando D, Basu S, Atkinson LP, Cao Y, Lee SF, Leeb M, Wohlfahrt KJ, Boucher W, O'Shaughnessy-Kirwan A, et al. 2017. 3D structures of individual mammalian genomes studied by single-cell Hi-C. Nature 544: 59-64.

Symmons O, Uslu VV, Tsujimura T, Ruf S, Nassari S, Schwarzer W, Ettwiller L, Spitz F. 2014. Functional and topological characteristics of mammalian regulatory domains. Genome Res 24: 390-400.

Tang Z, Luo OJ, Li X, Zheng M, Zhu JJ, Szalaj P, Trzaskoma P, Magalska A, Wlodarczyk J, Ruszczycki B, et al. 2015. CTCF-mediated human 3D genome architecture reveals chromatin topology for transcription. Cell 163: $1611-1627$.

Uuskula-Reimand L, Hou H, Samavarchi-Tehrani P, Rudan MV, Liang M, Medina-Rivera A, Mohammed H, Schmidt D, Schwalie P, Young EJ, et al. 2016. Topoisomerase II $\beta$ interacts with cohesin and CTCF at topological domain borders. Genome Biol 17: 182.

Vietri Rudan M, Barrington C, Henderson S, Ernst C, Odom DT, Tanay A, Hadjur S. 2015. Comparative Hi-C reveals that CTCF underlies evolution of chromosomal domain architecture. Cell Rep 10: 12971309.

Wang J, Li X, Wang L, Li J, Zhao Y, Bou G, Li Y, Jiao G, Shen X, Wei R, et al. 2016a. A novel long intergenic noncoding RNA indispensable for the cleavage of mouse two-cell embryos. EMBO Rep 17: 1452-1470.
Wang S, Su JH, Beliveau BJ, Bintu B, Moffitt JR, Wu CT, Zhuang X. 2016b Spatial organization of chromatin domains and compartments in single chromosomes. Science 353: 598-602.

Wen B, Wu H, Bjornsson H, Green RD, Irizarry R, Feinberg AP. 2008 Overlapping euchromatin/heterochromatin-associated marks are enriched in imprinted gene regions and predict allele-specific modification. Genome Res 18: 1806-1813.

Xiao R, Tang P, Yang B, Huang J, Zhou Y, Shao C, Li H, Sun H, Zhang Y, Fu XD. 2012. Nuclear matrix factor hnRNP U/SAF-A exerts a global control of alternative splicing by regulating U2 snRNP maturation. Mol Cell $\mathbf{4 5}$ : 656-668.

Ye J, Beetz N, O'Keeffe S, Tapia JC, Macpherson L, Chen WV, Bassel-Duby R, Olson EN, Maniatis T. 2015. hnRNP U protein is required for norma pre-mRNA splicing and postnatal heart development and function. Proc Natl Acad Sci 112: E3020-E3029.

Zuin J, Dixon JR, van der Reijden MI, Ye Z, Kolovos P, Brouwer RW, van de Corput MP, van de Werken HJ, Knoch TA, van IJcken WF, et al. 2014. Cohesin and CTCF differentially affect chromatin architecture and gene expression in human cells. Proc Natl Acad Sci 111: 996-1001.

Received May 1, 2017; accepted in revised form December 20, 2017.

\section{Genome Research}




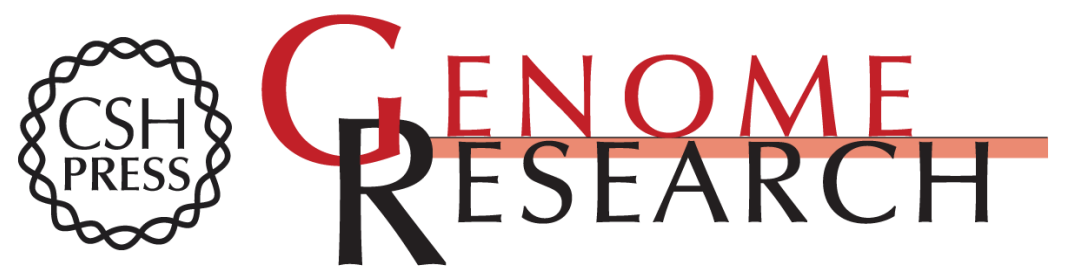

\section{The nuclear matrix protein HNRNPU maintains 3D genome architecture globally in mouse hepatocytes}

Hui Fan, Pin Lv, Xiangru Huo, et al.

Genome Res. 2018 28: 192-202 originally published online December 22, 2017

Access the most recent version at doi:10.1101/gr.224576.117

Supplemental Material

References

Creative

Commons

License

Email Alerting Service
http://genome.cshlp.org/content/suppl/2018/01/12/gr.224576.117.DC1

This article cites 65 articles, 20 of which can be accessed free at: http://genome.cshlp.org/content/28/2/192.full.html\#ref-list-1

This article is distributed exclusively by Cold Spring Harbor Laboratory Press for the first six months after the full-issue publication date (see

$\mathrm{http}: / /$ genome.cshlp.org/site/misc/terms.xhtml). After six months, it is available under a Creative Commons License (Attribution-NonCommercial 4.0 International), as described at http://creativecommons.org/licenses/by-nc/4.0/.

Receive free email alerts when new articles cite this article - sign up in the box at the top right corner of the article or click here.

\section{Affordable, Accurate Sequencing.}

To subscribe to Genome Research go to:

https://genome.cshlp.org/subscriptions 